\title{
LA GLOBALIZACIÓN DE LA EDAD DEL HIERRO EN EL MEDITERRÁNEO ${ }^{1}$
}

Tamar Hodos ${ }^{2}$

\section{Resumen}

La Edad del Hierro en el Mediterráneo (c. 1200-600 AEC) es uno de los períodos más dinámicos. Aunque no fue la primera era en la que sus habitantes intercambiaron mercancías, ideas, valores, costumbres, prácticas y tecnologías, la escala fue sin precedentes. El período se caracteriza, sobre todo, por el asentamiento griego y fenicio alrededor de todo el mar, lo que facilitó una amplia y frecuente comunicación. El impacto a largo plazo fue la creación del primer período conectado a escala global del Mediterráneo. Sin embargo, los académicos no nos han presentado, hasta ahora, una era globalizada. Este capítulo comienza con la definición de globalización y evalúa ocho tendencias fundamentales que se producen en la globalización contemporánea. Describe luego la Edad del Hierro del Mediterráneo y nuestros antecedentes académicos en la interpretación de los movimientos coloniales griegos y fenicios, desde la concepción colonial hasta la poscolonial. Por último, analiza cómo las teorías de la globalización contemporánea pueden transformar nuestro entendimiento de esta era culturalmente compleja y socialmente pujante.

\section{Palabras clave}

Edad del Hierro en el Mediterráneo; Globalización; Colonización; Hibridación; Colonialismo; Poscolonialismo.

\footnotetext{
${ }^{1}$ Traducción - Anabelle Silvana Luzardo Medeiros y Rosa Maria Severino Ueno.

${ }^{2}$ Assistant Professor - University of Bristol, Bristol, UK. E-mail: t.hodos@bristol.ac.uk

Heródoto, Unifesp, Guarulhos, v.4, n.1 - 2019.1. p. 74-102

DOI: https://doi.org/10.34024/herodoto.2019.v4.10088
} 


\section{Resumo}

A Idade do Ferro Mediterrânea (c. 1200-600 AEC) foi um dos seus períodos mais dinâmicos. Embora não tenha sido a primeira era na qual seus habitantes trocaram bens, ideias, valores, costumes, práticas e tecnologias, a escala foi sem precedentes. Em grande parte, o período é caracterizado por assentamentos gregos e fenícios ao redor de todo o mar, facilitando uma comunicação frequente e ampla. O impacto a longo prazo foi a criação do primeiro período globalmente conectado do Mediterrâneo. Entretanto, uma era globalizada nem sempre foi a narrativa apresentada pelos estudiosos. O presente capítulo começa definindo globalização e avaliando oito tendências fundamentais que ocorrem na globalização contemporânea. Depois, ele esboça a Idade do Ferro Mediterrânea e a nossa história acadêmica na interpretação dos movimentos coloniais gregos e fenícios, de perspectivas colonialistas até pós-coloniais. Finalmente, ele explora como as teorias de globalização contemporâneas podem transformar a nossa compreensão desta época culturalmente complexa e socialmente vibrante.

\section{Palavras-Chave}

Idade do Ferro Mediterrânea; globalização; colonização; hibridização; colonialismo; pós-colonialismo. 


\section{Cómo definir la globalización}

La globalización es uno de los procesos más importantes de integración económica, social y cultural en el mundo actual e incluye el comercio, el capital y las inversiones, la migración laboral y la difusión de conocimientos. Muchos dirían que se concreta mediante el libre comercio y el capitalismo (Cf. Steiglitz, 2006: 3-24; Ward, England, 2007: 1-22; Pieterse, 2014: 1-11). Pero la globalización es más que apenas una forma de describir el neoliberalismo contemporáneo. De hecho, no hay consenso en cuanto a la definición de globalización, aunque muchos caracterizan que la creciente conectividad es su rasgo más ampliamente aceptado (Cf. Giddens, 1990; Robertson, 1992; Moore, Lewis, 2009). Esta conectividad es particularmente de un tipo que abarca un vasto flujo de ideas y conocimientos, junto con el intercambio de costumbres culturales y prácticas de la sociedad civil. Estos flujos y conexiones pueden manifestarse a través de una integración económica más estrecha, mediante el aumento de la circulación de bienes y servicios, capital y mano de obra, o pueden estar determinados por la política. (Steiglitz, 2006: 05)

Sin embargo, muchas características del debate relativo a la globalización, tales como si esta se basa en los cambios tecnológicos, si implica la reconfiguración de estados, etc., se dan la mano con la regionalización o incluyen un sentido de la compresión tiempo-espacio (Cf. Pieterse, 2015: 725). La globalización suele describirse en un marco disciplinario particular, de manera que escuchamos acerca de diferentes tipos de globalización, entre ellos la globalización financiera, comercial, económica o política. La mayoría de las personas, en todo caso, está de acuerdo en que la globalización es desigual y asimétrica en el ritmo, el alcance y el impacto que ejerce. Los estudiosos entienden estos acontecimientos ya sea como un proceso (entre ellos Robbie Robertson y Jan Nederveen Pieterse), como un sistema (por ejemplo, Jonathan Friedman, Immanuel Wallerstein) o bien como un discurso (como Manfred Steger). ${ }^{3}$

El sociólogo cultural Tomlinson define la globalización simplemente como conectividades complejas (Tomlinson, 1992: 02). El sociólogo Roland Robertson, por otro lado, define la globalización como el proceso mediante el cual el mundo es visto, cada vez más, como un solo lugar y las formas por las cuales nos hacemos conscientes de ese proceso (Robertson, 1992: 08), aunque, como el sociólogo Mike Featherstone ha señalado, esto no debería interpretarse en el sentido de que existe una cultura mundial

\footnotetext{
${ }^{3}$ Nederveen Pieterse, Globalization and Culture, Cuadros 1.6 y 1.7, 19-20, con referencias en 183-210.
}

Heródoto, Unifesp, Guarulhos, v.4, n.1 - 2019.1. p.

DOI: https://doi.org/10.34024/herodoto.2019.v4.10088 
unificada (Featherstone, 1995: 114). Por esta razón, los estudios globales y el estudioso de la sociología Jan Nederveen Pieterse definen la globalización como "un proceso de hibridación que da lugar a una mezcla global" (Pieterse, 2015: 67), y argumenta que la globalización puede ser entendida como una síntesis abierta de una serie de enfoques disciplinarios a esos acontecimientos, en el que hay tantos modos de globalización como agentes, dinámicas e impulsos (Pieterse, 2015: 68).

En otro lugar he definido la globalización como "procesos de conectividades crecientes que se despliegan y se manifiestan como conciencia social de esas conectividades" (Hodos, 2017: 03-11, 04). He argumentado que debido a que la globalización tiene que ver con los procesos en sí, es un concepto activo más que descriptivo. También he observado que la idea sugiere una escala mundial y que ese es el motivo de que algunos insistan en que se trata de un fenómeno que solo comienza con la aparición de la circunnavegación global (Cf. Giddens, 1990; Wallerstein, 1991; Robertson, 2003; Hodos, 2017: 54-65). Se utiliza con mayor frecuencia, sin embargo, para reflejar cambios más amplios en el interior de un mundo conceptual o experimentado. La mayoría de las veces, implica una creciente integración y cooperación derivada de las siempre cambiantes prácticas comunes que facilitan dicha integración y cooperación (Hodos, 2017: 04). Con esto no se quiere sugerir que la conectividad y el conocimiento de estas conexiones, por sí solas, representen un período o lugar globalizado. Las contribuciones al reciente Routledge Handbook of Archaeology and Globalization demuestran que la naturaleza de las complejas conexiones sociales y la manera en que la conciencia de las mismas se manifiesta son las que determinan si una época en el pasado puede considerarse globalizada o no (Hodos, 2017).

Un corolario importante de la creciente sensación de similitud, accesibilidad y comunalidad que asociamos con la globalización es la conciencia, e incluso el aumento, de las diferencias y las desigualdades más pronunciadas con aquellos no tan estrechamente integrados en el ámbito experiencial, o con quienes no participan en absoluto. Esto indica que los procesos de globalización presentan dos aspectos fundamentales: 1. El desarrollo de prácticas compartidas y valores que contribuyen al sentimiento de pertenencia al mismo lugar; 2. Mayor conciencia y sensibilidad a las diferencias, especialmente a las culturales. Ambos aspectos derivan de una variedad de crecientes conectividades, aunque normalmente es el primero el que recibe más atención. No obstante, el aumento de la conciencia de la diferencia cultural es una función de la globalización, no solo una característica de la misma. Esto se manifiesta

Heródoto, Unifesp, Guarulhos, v.4, n.1 - 2019.1. p.

DOI: https://doi.org/10.34024/herodoto.2019.v4.10088 
generalmente como un resurgimiento de las prácticas de identidad local en contraste explícito con las prácticas cada vez más compartidas de lo globalmente conectado. Estas expresiones locales de la identidad suelen vincularse también con niveles muy divergentes de riqueza, salud y poder político. Como resultado, estos acontecimientos generalmente aumentan el contraste entre las partes involucradas en el sistema global y el grado de inversión social en el mantenimiento de la diferencia cultural. La globalización, por lo tanto, mantiene un equilibrio entre las prácticas compartidas y vinculantes y las diversidades que distinguen a los participantes. Ellas no solo están en tensión una con la otra, sino también son interdependientes y juntas crean la paradoja de la globalización (Hodos, 2017: 05). La globalización no puede ser conceptualizada ni examinada adecuadamente sin tener en cuenta ambos aspectos. Sin embargo, muchas discusiones académicas y populares acerca de la globalización reconocen solo el primero e ignoran el segundo.

\section{Cómo identificar la globalización}

El arqueólogo Justin Jennings identifica ocho tendencias que se producen en la globalización contemporánea, (Cf. Jennings, 2011: 123-141; Jennings in: Hodos, 2017: 12-28, 14-16), las cuales se superponen y son estimuladas por conectividades complejas para crear una cultura global. Sostiene que todas deben estar presentes en cualquier período para que este pueda ser considerado una era de globalización.

(1) la compresión tiempo-espacio. Identificada por el geógrafo David Harvey (1989), es la experiencia de que nuestro mundo se reduzca mediante la aceleración de los procesos de larga distancia, económicos, políticos y sociales, de tal forma que los cambios en un lugar se puedan ramificar en una amplia región. Internet es un ejemplo que contribuye a nuestro moderno sentido de la compresión tiempo-espacio, las repercusiones de la crisis económica del 2008 en todo el mundo occidental son otro. En el pasado, la introducción de un nuevo animal gregario o la adopción de un sistema de grabación produjeron efectos similares.

(2) la desterritorialización. Es la sensación de que un lugar tiene una conexión menos estrecha con su contexto local y geográficamente establecido. Se produce tanto a través de la extensión de las redes sociales como de la incorporación de personas, bienes, ideas y prácticas extranjeras en un entorno local. Actualmente, uno de los efectos de la desterritorialización es el cosmopolitismo de nuestras denominadas

Heródoto, Unifesp, Guarulhos, v.4, n.1 - 2019.1. p.

DOI: https://doi.org/10.34024/herodoto.2019.v4.10088 
ciudades internacionales en todo el mundo, para las cuales los vínculos con una única ubicación fija disminuyen debido a la multitud de interacciones de larga distancia que conectan esos lugares con otras ciudades y regiones en el resto del mundo. De esta manera, París, como centro cosmopolita de la moda, la cultura, el arte y la gastronomía, tiene poco que ver con su ubicación geográfica, y en ese sentido se conecta mucho menos con sus alrededores que con otros centros cosmopolitas de la moda, la cultura, el arte y la gastronomía, como Nueva York, Londres o Tokio. En el pasado, la cantidad y la variedad de los estilos importados de productos materiales en ciertas épocas fomentó la misma impresión.

(3) la normalización/estandarización/uniformización. Esto se desarrolla a medida que las personas navegan, lo que significa superar las divisiones geográficas y socioculturales. Los estándares de medición universales, como la hora del Meridiano de Greenwich o el sistema métrico, son ejemplos que usamos hoy en día. En el pasado, los lenguajes administrativos ayudaron a promover la normalización en vastos territorios constituidos por diversos grupos culturales.

(4) la desigualdad La globalización no es un proceso ecuánime en términos de inclusión. Esto se debe a que las redes de interacción interregional no son geográficamente ubicuas. Como resultado, pueden haber considerables diferencias de poder entre las regiones que participan. Así, China, por ejemplo, domina actualmente muchas relaciones de intercambio mientras que otros países siguen estando relativamente aislados, a pesar de su participación en los intercambios mundiales, como Haití. Bollywood goza de una influencia global, pero la industria cinematográfica iraní no. Con respecto al pasado, esto se ve en cómo una ciudad podía disfrutar de mucha mayor influencia que otros lugares de su época y en la dispersión en la propagación de horizontes estilísticos.

(5) la homogeneización. Este es quizá el distintivo más ampliamente discutido de los procesos de globalización y ante el cual las personas y los medios de comunicación más reaccionan. Se produce un grado de homogeneización cultural cuando los pueblos comienzan a compartir un conjunto similar de prácticas y productos para gestionar una creciente circulación de ideas, objetos y personas. No obstante, no se trata tanto de una única forma de vida. Por el contrario, se refiere a cómo podemos entrar en contacto con ideas y bienes ampliamente compartidos y cómo nos apropiamos de ellos en nuestros contextos locales. Una visita a McDonalds en Europa o Asia, por ejemplo, puede ser al comienzo una aventura novedosa y exótica, pero con el tiempo se incorpora tanto a las prácticas locales que pasa a ser autóctona y deja de ser vista como un emblema

Heródoto, Unifesp, Guarulhos, v.4, n.1 - 2019.1. p.

DOI: https://doi.org/10.34024/herodoto.2019.v4.10088 
extranjero (Caldwell, 2004: 05-26). La conformación de actitudes entre las prácticas y los valores sociales, como con respecto a la belleza o la violencia, son otros ejemplos contemporáneos. En el pasado, esto se establece mediante prácticas convergentes entre diversos grupos, tales como el uso de objetos y estilos similares.

(6) la heterogeneidad cultural. Esto ocurre porque, junto con el incremento de la homogenización, la variación cultural a menudo aumenta al mismo tiempo, especialmente a medida que las prácticas se reinterpretan a nivel local. La mezcla de influencias externas con las prácticas locales varía de un lugar a otro. Así, el yoga se reinterpreta en los estudios estadounidenses y las hamburguesas en Japón no son exactamente lo que uno podría reconocer como tal en el mundo occidental. En el pasado, esto se ejemplifica mejor mediante las variabilidades de cerámica visual que combinan una serie de ideas.

(7) reincorporación de la cultura local. Puede comprenderse como el resurgimiento, o incluso la invención, de las prácticas y formas locales en reacción explícita a la homogenización promovida por la creciente globalización. La Académie francesa mantiene la pureza de la lengua francesa en nuestros días, por ejemplo. En el pasado, se encontraba en la reiteración de las tradiciones locales, tales como los tipos de funerales, la organización de los asentamientos y otras prácticas.

(8) la vulnerabilidad. Las cuantiosas conexiones profundamente arraigadas entre los diversos grupos que crean una circunstancia de compleja conectividad, también conducen a la interdependencia y, por consiguiente, al riesgo. La conciencia de los riesgos de la globalización se agudiza cuando se produce un evento que resuena a través de la red. Los desafíos enfrentados debido a la desvinculación de Gran Bretaña de la Unión Europea, que continuarán mucho después de que Gran Bretaña haya salido, ponen de relieve la vulnerabilidad de las complejas conexiones actuales que han sido creadas por la red globalizada que es la Unión Europea. En el pasado, cuando los grandes centros como Roma fracasaron, el sistema de intercambio globalizado que había creado alrededor de sí misma se dividió en redes menores que operaban a una escala más restringida.

Jennings afirma que todos estos elementos deben estar presentes para identificar la globalización. Pero, ¿qué los define como presentes? Casi todas estas ocho características son, de hecho, fáciles de encontrar en el pasado en formas comparables con nuestras experiencias actuales, aunque algún aspecto resulte más difícil que otro, precisamente debido a la forma

Heródoto, Unifesp, Guarulhos, v.4, n.1 - 2019.1. p.

DOI: https://doi.org/10.34024/herodoto.2019.v4.10088 
de percepción que tenemos hoy. Esta es la idea de la compresión tiempoespacio. Los recientes avances tecnológicos han hecho que el mundo de hoy esté conectado de manera más fácil y rápida. Nuestra capacidad de comunicarnos en todo el mundo hace que el tiempo y el espacio se perciban como comprimidos en una experiencia inmediata y ampliamente compartida, de manera tal que el mundo se percibe hoy por hoy más pequeño que hace una generación. Puesto que existe poca evidencia de un aumento tan drástico en las comunicaciones en el pasado, algunos sostienen que la globalización, por lo tanto, solo puede ser un fenómeno muy moderno y cuestionan su aplicación al pasado passado (Cf. Morley in: Pitts e Versluys, 2014: 49-68). Otros, que se centran en su escala geográfica, sugieren que no puede ser anterior al siglo XVI y a la circunnavegación del mundo (Cf. Giddens, 1990; Wallerstein, 1991; Robertson, 2003; Hodos, 2017: 54-65), anunciando la llegada de la era de los descubrimientos europeos, aunque esto favorece los conceptos occidentales en nuestros entendimientos de la globalización.

La compresión tiempo-espacio en sí puede ser impugnada por la fenomenología, el estudio de las estructuras de la experiencia y de nuestra conciencia de la experiencia, dado que el mundo no está físicamente reduciéndose ni contrayéndose como resultado de la globalización. La impresión se deriva de la comunicación más rápida entre los grupos, que ha creado la sensación de que el mundo es más pequeño, ya que comunicarse es menos demorado que antes. En otras palabras, la experiencia cultural de la globalización para la mayoría de los pueblos no se deriva de su propia movilidad directa.

Dado que no estamos experimentando de hecho la compresión de tiempo y espacio, ¿qué es lo que estamos experimentando? Uno podría argumentar que se trata de un sentimiento de pertenencia al mismo lugar (Hodos in: Pitts e Versluys, 2014: 240-253). Podemos comunicarnos con alguien del otro lado del mundo en un instante, como si estuviéramos en el mismo sitio. Anteriormente, la llegada del teléfono hizo posible hablar directamente con alguien que estaba lejos. Las redes de larga distancia de rutas, caminos y senderos servían al mismo propósito en el pasado más distante. Hicieron que los lugares lejanos se sintieran conectados, al igual que las rápidas comunicaciones lo posibilitan hoy por hoy. Pero hay otras formas de transmitir esta conexión, este sentimiento de pertenencia al mismo lugar. En la antigüedad, la mayoría de la gente no viajaba muy lejos, o no podía viajar en absoluto. En cambio, las redes de prácticas compartidas transmitieron esta noción de pertenencia al mismo lugar. En el mundo romano, por ejemplo, la velocidad de los viajes y de las

Heródoto, Unifesp, Guarulhos, v.4, n.1 - 2019.1. p.

DOI: https://doi.org/10.34024/herodoto.2019.v4.10088 
comunicaciones no aumentaba significativamente por todo el Imperio Romano cuando una región se incorporaba a él, a pesar de la construcción de redes viales (Cf. Morley in: Pitts e Versluys, 2014: 49-68). Pero hubo un aumento de la frecuencia de las comunicaciones, lo que condujo a una mayor información sobre las regiones, rutas y precios, así como a más circulación regular de los productos y servicios compartidos. Esto dio lugar a la generalización de mercancías y prácticas, lo que creó un sentimiento de pertenencia al mismo lugar. Era a través de las redes de prácticas y bienes en común que las personas vivían el sentido de compresión del tiempo y del espacio en una experiencia compartida y vital.

\section{La Edad del Hierro en el Mediterráneo}

A pesar de su apodo, la era de la Edad del Hierro en el Mediterráneo no está estrictamente correlacionada con el desarrollo de la metalúrgica, dado que el hierro ya era conocido en los milenios quinto y cuarto AEC en Irán (Sialk), Irak (Samarra) y Egipto (Gerzense) y, en el tercer milenio en Mesopotamia y Anatolia (Tilmen Höyük; Alaca Höyük) ) (Waldbaum, 1978; Waldbaum in: Piggot, 1999: 27-57; Yalçın, 1999: 177-187). No obstante, el término "Edad del Hierro" se considera mejor como un medio de marcar cambios con tradiciones y prácticas asociadas con sus antecedentes temporales. Lo que siguió fueron los cambios en las prácticas sociales, que se manifiestan en los restos materiales a través,

principalmente, de nuevas formas y estilos de cerámica, las formas arquitectónicas y las prácticas funerarias. Tales cambios no ocurrieron en una sola instancia, sino que se desarrollaron a lo largo del tiempo, y la tasa de cambio dentro de cualquier categoría material no era uniforme de un sitio a otro o de una región a otra. El concepto de la Edad del Hierro es, por lo tanto, un concepto relacional.

En el Mediterráneo, esta ruptura se produce a fines del siglo XII AEC, que se considera el final de la Edad del Bronce. Esto trajo como consecuencia la muerte definitiva del sistema palacio micénico en el Egeo, la desintegración del imperio hitita en Anatolia y Mesopotamia, la invasión de Egipto y la costa del Mediterráneo oriental por los llamados Pueblos del Mar y el final de la era del Nuevo Reino de Egipto. El contacto generalizado entre estas comunidades disminuyó drásticamente, especialmente en comparación con la cantidad de bienes, ideas y personas circulantes, que precedió y sucedió a los primeros siglos del primer milenio AEC. Colectivamente, esto forma parte de lo que crea una sensación general de cambio y lo que marca el inicio de la Edad del Hierro en el Mediterráneo.

Heródoto, Unifesp, Guarulhos, v.4, n.1 - 2019.1. p.

DOI: https://doi.org/10.34024/herodoto.2019.v4.10088 
Lo que más caracteriza a la Edad del Hierro en el Mediterráneo es la circulación de personas, que se destacan por la cantidad y por su naturaleza. Por el siglo IX AEC, si no antes, individuos y pequeños grupos comenzaron a desplazarse alrededor del Mediterráneo. Algunos de ellos eran vendedores ambulantes y artesanos, pero también se aprecia la circulación de grandes grupos de personas que residían en las costas extranjeras. Los dos movimientos con mayor impacto son los fenicios y los griegos.

A pesar de que tanto los griegos como los fenicios fundaron colonias de ultramar por todo el Mediterráneo y a veces en el mismo territorio geográfico (p. ej. Sicilia), el estudio de su expansión ha estado dividido entre disciplinas (Hodos, 2009: 221-241). Los estudiosos del Cercano Oriente tradicionalmente se centraron en el proceso de colonización fenicia, mientras que los estudiosos clásicos examinaron la circulación griega. Esta división disciplinaria puede remontarse al estudio europeo occidental del siglo XIX y a la respectiva primacía de antiguas fuentes literarias clásicas y bíblicas, atribuidas por la investigación arqueológica en esa era. A menudo esto se expresa a través de una competencia por la primacía en la innovación colonial. Las preguntas sobre las fechas de fundación de las primeras colonias, enmarcadas como si una supremacía de innovación dependiera de ello, son síntomas de esta rivalidad. La mayor parte de las fuentes literarias antiguas nos dicen que la expansión fenicia en el Mediterráneo se inició en el siglo XII AEC, mientras que la colonización griega comenzó recién en el siglo VIII (Cf. Hodos, 2009: 221241; Pieterse, 2015: 7-25). Durante mucho más de un siglo, sin embargo, la academia griega argumentó que la expansión fenicia no podía haber comenzado antes del siglo VIII (Beloch, 1913: 229-232), alegando que no podían identificarse pruebas materiales que apoyaran esas primeras fundaciones en lugares donde los fenicios supuestamente colonizaron. Por ejemplo, John Boardman ha sostenido que

Solo después de que los griegos se establecieron en la costa siria Grecia comenzó a recibir y a apreciar los productos orientales; y no hay evidencias claras de la existencia de colonias comerciales fenicias de ultramar antes que las griegas. Por todo ello, pueden haber sido los portadores de lo poco que sí viajó al mundo griego desde oriente antes del siglo VIII. La naturaleza de este comercio no requiere el establecimiento de puestos comerciales regulares ni colonias hasta que el ejemplo de Grecia y la competencia los condujo a empresas similares. (Boardman, 1999: 38).

Más recientemente, Gocha Tsetskhladze observó que:

En el siglo VIII A.C., los griegos se desplazaban hacia territorios relativamente cercanos del centro y del sur de Italia, mientras que los fenicios establecieron

Heródoto, Unifesp, Guarulhos, v.4, n.1 - 2019.1. p.

DOI: https://doi.org/10.34024/herodoto.2019.v4.10088 
pequeños asentamientos en Cerdeña y hacia el oeste y el sur. Los asentamientos griegos se concibieron para permanecer, los de los fenicios desaparecieron con el tiempo, probablemente absorbidos por los lugareños (Tsetskhladze in: Tsetskhladze, 2006: 23-83, 49).

Esta declaración presenta un enfoque limitado y objetivamente incorrecto, dado que ignora los importantes y permanentes asentamientos fenicios de Sicilia, España y África del Norte, así como las comunidades griegas contemporáneas que no sobrevivieron. Las fundaciones fenicias no eran menos "permanentes" que las griegas. De hecho, la discusión de un determinado territorio suele basarse en términos de prioridad griega o fenicia. Los académicos tienden a prestar escasa atención a la posibilidad de que un mismo entorno pueda haber sido compartido por ambos grupos, o cualquier otro pueblo, a pesar del hecho de que los demás ya estaban establecidos en los territorios a los que llegaron los griegos y los fenicios. ${ }^{4}$

La academia fenicia ha hecho lo mismo en gran medida. Por ejemplo, Sabatino Moscati argumentó casi a la defensiva, en su trabajo seminal sobre el tema, que la falta de pruebas materiales anteriores al siglo VIII (como era el caso hasta hace muy poco) no era motivo para dudar de los textos griegos y bíblicos y tampoco de los registros fenicios que demuestran la actividad fenicia mucho antes (Moscati, 1966). La dificultad surge del hecho de que la cerámica es nuestro principal medio de datar sitios arqueológicos y contextos durante este período. El problema con las pruebas de asentamientos fenicios, sin embargo, es que la cerámica fenicia sufre muy pocos cambios de estilo sobre el final del segundo y primer milenio y carece de la fina precisión cronológica de la cerámica griega. Así que cuando se encuentra material cerámico fenicio, puede ser muy difícil determinar exactamente su fecha. Por el contrario, casi en todos los lugares donde los griegos se asentaron, sus utensilios para beber vino y otros artefactos, con datación muy cercana, rápidamente se integraron a los gustos y a las costumbres locales y sus ensambles de cerámica influyeron en la producción autóctona regional. Además, muchos asentamientos adoptaron las formas arquitectónicas derivadas de los griegos. Aunque originalmente derivado del fenicio, el alfabeto griego se adaptó a veces para expresar las lenguas locales y muchas comunidades en algún momento tomaron prestado el sistema griego de acuñación. En tanto que los fenicios eran tan frecuentes en el Mediterráneo, su cultura material no fue ampliamente adoptada por otros. De hecho, el uso de cerámica griega y las ideas arquitectónicas fueron incorporados por los fenicios a sus prácticas y construcciones urbanas.

${ }^{4}$ Cf. (Boardman, 1999: 213) en lo que respecta a España.

Heródoto, Unifesp, Guarulhos, v.4, n.1 - 2019.1. p.

DOI: https://doi.org/10.34024/herodoto.2019.v4.10088 
Inicialmente, esta prevalencia de formas culturales griegas se interpretó como un deseo de ilustración cultural y sofisticación de las comunidades autóctonas (y de los fenicios, hasta cierto punto, que fueron en gran parte ignorados porque formaban parte de los estudios del Cercano Oriente). Quien mejor lo resumió fue Boardman en la descripción que realizó sobre la interacción con las poblaciones no extranjeras de Sicilia e Italia, cuando afirma que: "En el occidente, los griegos no tenían nada que aprender, sino mucho que enseñar." (Boardman, 1999: 190). En este modelo de "helenización", los griegos mantienen su hegemonía cultural, social, política y económica. Permanecen inmunes ante cualquier influencia por parte de aquellos que fueron helenizados y que adoptaron la cultura helénica en forma generalizada e incondicionalmente. Se tienen muy poco en cuenta la intermediación o la reciprocidad. La helenización es, pues, un proceso binario y unidireccional. Esta interpretación dominó nuestros puntos de vista durante gran parte del siglo XX, desde el período de entreguerras, cuando las colonias griegas eran consideradas gloriosas contrapartes a las posesiones coloniales de Europa.

A comienzos de los años noventa, sin embargo, la arqueología comenzó a verse influenciada por la deconstrucción posmoderna que se inició en el amplio discurso intelectual de occidente durante la década de 1980, especialmente la aparición del poscolonialismo, resultante del trabajo de Edward Said y Gayatri Spivak. En particular, dichos argumentos en contra, como las deconstrucciones de las metanarrativas del discurso colonial, inspiraron a los estudiosos de la arqueología a reconsiderar sus interpretaciones de las colonizaciones griega y romana explorando su impacto desde el punto de vista de los colonizados (Cf. Hodos, 2014: 2430). Exámenes detallados del uso y la práctica de la cultura material entre los pueblos autóctonos reveló que la adopción generalizada de los bienes y las prácticas extranjeras no se estaba produciendo. Por ejemplo, se observó en diferentes territorios, como Sicilia, África del Norte y Francia, que las comunidades autóctonas eran muy selectivas en lo que elegían adquirir de sus vecinos extranjeros, en particular con respecto a determinadas formas de cerámica o características arquitectónicas. Además, el índice de absorción podría producirse decenios o incluso siglos después de la llegada de los griegos a una región (Cf. Dietler, 2010; Hodos, 2006). Una interpretación de simple helenización no correspondía a tal evidencia. Por el contrario, estos ejemplos se ajustan mejor a un modelo de interpretación de hibridación. Cuando se conecta con la práctica cultural, la hibridación puede ser considerada como la mezcla cultural que surge de las prácticas de orígenes mixtos (Friedman in: Werbner e Modood, 1997: 70-89, 88; Burke, 2009: 34). Las personas y los objetos materiales en tales

Heródoto, Unifesp, Guarulhos, v.4, n.1 - 2019.1. p.

DOI: https://doi.org/10.34024/herodoto.2019.v4.10088 
situaciones crean nuevas prácticas sociales y materiales. Estas prácticas suelen combinar tanto las formas coloniales como las tradicionales, aunque serán una mezcla única para el grupo en cuestión. En resumen, la hibridación es un proceso activo relacionado con los agentes sociales y sus negociaciones e interacciones en situaciones de contacto cultural. Es también un proceso localmente contextualizado y específico en el tiempo.

Las interpretaciones inspiradas en el poscolonialismo de la Edad del Hierro en el Mediterráneo que se centran en las experiencias de los pueblos colonizados han llegado a ser acusadas de no pasar de ejemplos de corrección política. Así, en la edición de 1999 de The Greeks Overseas, Boardman se pregunta si el reciente énfasis en las experiencias de los colonizados es simplemente parte de un "empuje [de] las normas modernas deseadas hacia la antigüedad y de hacer suposiciones acerca de los prejuicios de las últimas generaciones de estudiosos" (Boardman, 1999: 268). No es el único. (Cf. Domínguez, 2012: 205-218). Estas críticas reflejan una insatisfacción con el cambio de énfasis porque el resultado parece a veces borrar al colonizador de la narrativa, especialmente cuando la adaptación se lleva a cabo en el largo plazo. No obstante, en realidad los trabajos poscoloniales nunca negaron que las culturas coloniales ejercieron un impacto profundo en los denominados pueblos colonizados. Más bien, el discurso poscolonial ha querido enfatizar explícitamente los actos de la intermediación por parte de los colonizados y destacar que los cambios que se produjeron en las poblaciones locales presentaban matices mucho más profundos que la simple adopción generalizada de las formas de vida colonial. Sin embargo, hay casos donde la escala temporal de adaptación sugiere que el origen explícitamente griego de las formas o prácticas quedó perdido en el tiempo, de tal forma que la utilización de esos estilos o costumbres ya no puede guardar ninguna asociación significativa con las tradiciones helénicas.

A finales de las décadas de 1990 y 2000, las reacciones al marco de interpretación helénica habían tomado dos trayectorias claramente definidas (Hodos, 2014: 27). La primera fue el énfasis en las diversidades de "ser griego". Esta veía la deconstrucción de la noción de que las colonias griegas eran réplicas de sus ciudades madre, y los académicos hacían hincapié en sus diversidades Cf. Dougherty e Kurke, 2003; Lomas, 2004; Tsetshkladze, 2006). También condujo a desafíos relacionados con un concepto unificado de "los griegos" como entidad cultural (Hall, 2002). He argumentado, sin embargo, que hay una cantidad suficiente de características comunes que aún nos permiten discutir una noción colectiva de los griegos (Hodos, 2009). El segundo énfasis planteó el papel

Heródoto, Unifesp, Guarulhos, v.4, n.1 - 2019.1. p.

DOI: https://doi.org/10.34024/herodoto.2019.v4.10088 
de las poblaciones autóctonas en la relación griegos - no griegos. Estudios han demostrado que el uso de cualesquiera características griegas, ya sea la cerámica, la arquitectura, las diferentes formas de funerales, la escritura $\mathrm{u}$ otras, fue gradual y selectivo, de una manera apropiada a las circunstancias locales (Cf. Dietler, 2010; Hodos, 2006).

El mejor ejemplo fenicio de este cambio de paradigma se ve en las interpretaciones del papel del tofet, recintos ceremoniales al aire libre donde se realizaba el sacrificio y entierro de niños como parte de las prácticas religiosas de los fenicios. Se han encontrado tofets principalmente en las colonias occidentales, aunque también se identificaron ejemplos del Cercano Oriente más allá de los territorios fenicios (p. ej. Tell Sukas). A pesar de ello, se ha asumido de todas maneras que el tofet refleja las prácticas de suelo natal. Moscati estaba convencido de ello al observar que "no hay pruebas de que estos lugares sagrados en Fenicia hayan existido, pero no hay dudas acerca de su existencia, si sumamos a la evidencia bíblica las sobradas pruebas proporcionadas por las excavaciones en las colonias occidentales," (Moscati, 1968: 77) adhiriéndose a la noción de colonia como réplica de la ciudad madre en el amplio mundo fenicio. Hoy por hoy, sin embargo, enfoques mucho más flexibles reconocen la variabilidad con respecto a la función del tofet, y las variabilidades se consideran como importantes para las circunstancias locales, en vez del reflejo de interpretaciones equivocadas de prácticas ampliamente compartidas (Aubet, 2001: 250-256; Quinn, 2018: 91-112). Esta palabra nunca aparece en la documentación fenicia o púnica, así que nuestra comprensión de la función y del significado de lo que llamamos tofets en en los contextos fenicios/púnicos es completamente ética. Era un santuario como otros tantos, donde el pueblo podía realizar transacciones con los dioses. Pero existía una tremenda variabilidad en los métodos funerarios y conmemorativos en los contextos del tofet. Los últimos análisis revelan que el prestigio social se destaca en la colocación de stelai. La presencia del propio tofet también se asocia con otras características de la urbanización en la medida en que puede considerarse como la primera expresión del carácter urbano del asentamiento, donde contribuyó tanto a lo cívico como a las comunidades territoriales (Quinn in: Xella, 2013: 23-48). En suma, el pensamiento actual es que el tofet es una etiqueta genérica que engloba diferentes realidades arqueológicas y conceptuales en todo el mundo fenicio, en lugar de un elemento puramente distintivo de lo fenicio-púnico. Jo Crawley Quinn realizó recientemente esta precisa observación: "si hubo una cultura institucional diáspora fenicia o púnica, los santuarios tofet no formaban parte de ella" (Quinn in: Xella, 2013: 40).

Heródoto, Unifesp, Guarulhos, v.4, n.1 - 2019.1. p.

DOI: https://doi.org/10.34024/herodoto.2019.v4.10088 
Desde nuestros antecedentes académicos, nos encontramos con varios juegos de pruebas que han creado perspectivas de polarización: las prácticas compartidas que dieron origen a conceptos como la helenización en primer lugar, la variabilidad de la práctica de griegos y fenicios y el resurgimiento de las prácticas locales frente a la propagación y el impacto de las culturas helénica y fenicia. Cada una tiene mérito en sí misma, como lo han demostrado las generaciones de estudiosos del pasado y del presente. La cuestión tiene que ver con una escala de perspectivas. Por un lado, sigue habiendo una adopción abrumadora de la cultura material griega, en particular, en una amplia zona. Por otro lado, si lo consideramos desde una perspectiva local, el uso de tales objetos es variable y significativo a nivel local. Por lo tanto, ¿cómo puede rechazarse una interpretación en favor de otra? Para recuperar estas perspectivas aparentemente opuestas en una narrativa complaciente, los estudiosos recurren cada vez más a las teorías de la globalización (Cf. Vlassopoulos, 2013; Sherratt in: Hodos, 2017: 602-617; Dommelen in: Hodos, 2017: 618633; Müller, 2016).

\section{¿Una Edad del Hierro Mediterránea globalizada?}

Volvamos a los ocho criterios de Jennings sobre la globalización y veamos si podemos identificarlos en la Edad del Hierro en el Mediterráneo, conectados por redes de colonias griegas y fenicias que enlazaron el norte y el sur, el este y el oeste. Aunque podríamos elegir muchos ejemplos que ilustran las ocho características, presento aquí solo una breve selección. La mayoría se centra en Sicilia, donde los griegos y fenicios coexistieron, también junto a las comunidades autóctonas. La isla presenta así uno de los mejores medios de examinar conectividades complejas en el antiguo Mediterráneo. No obstante, también se ofrecen breves comparaciones con otras regiones.

En primer lugar, tenemos la compresión tiempo-espacio. Para la Edad del Hierro en el Mediterráneo, diría que tenemos un sentido de compresión tiempo-espacio. El establecimiento de asentamientos griegos y fenicios alrededor del Mediterráneo permitió la comunicación regular y frecuente en toda la cuenca mediterránea. Dicha comunicación, por necesidad, fue una mezcla de comunicaciones de larga distancia y localizadas, cabotaje, travesías por mar. ${ }^{5}$ Fue a través de estos asentamientos que la cultura

\footnotetext{
${ }^{5}$ La énfasis de Horden, Purcell, Corrupting Sea. Para observar un estudio de caso, véase Justin Leidwanger, "Modeling distance with time in ancient Mediterranean seafaring: a GIS application for the interpretation of maritime activity," Journal of Archaeological Science 40.8 (2013): 3302-08.
}

Heródoto, Unifesp, Guarulhos, v.4, n.1 - 2019.1. p.

DOI: https://doi.org/10.34024/herodoto.2019.v4.10088 
material griega y fenicia se propagó tan extensamente por todo el Mediterráneo. A continuación se abordará el hecho de que la cultura material griega fue ampliamente adoptada. El punto es que los propios asentamientos crearon nodos en el mercado, dado que esos colonos adquirían mercancías y objetos culturalmente familiares para uso propio y para el intercambio con las comunidades vecinas, que pueden haber pertenecido a diversas culturas. La naturaleza permanente de estos asentamientos introdujo, además, una cultura material no móvil, como los estilos arquitectónicos y las características culturales no materiales, entre ellas las prácticas sociales y religiosas, a estos otros, cuyos elementos también se adoptaron posteriormente. Colectivamente, estos elementos crearon un sentimiento de pertenencia a lo largo del Mediterráneo.

En contraste, durante la Edad del Bronce, las interacciones regulares y frecuentes del tipo que crea un sentimiento de pertenencia al mismo lugar se centraron mucho más en torno al mar Egeo (Knappet, Evans e Rivers, 2008: 1009-1024; Knappet, Evans e Rivers, 2011: 1008-1023). Por su naturaleza, eran generalmente de menor duración. Si bien hay pruebas de intercambio a una distancia más larga, como entre el mundo micénico y Sicilia (Leighton, 1999: 170-180; Wijngaarden, 2002), estas parecen ser irregulares y excepcionales. Por lo tanto, es recién durante la Edad del Hierro que podemos hablar de un sentimiento de compresión tiempoespacio a lo largo y a lo ancho del Mediterráneo (Sherratt in: Hodos, 2017: 602-617).

La segunda es la desterritorialización. En el transcurso de los siglos IX y VIII, la cantidad y variedad de artículos en circulación en todo el Mediterráneo - mucho más que en contextos puramente de élite - sugiere la evolución del comercio. Vinos, aceites, metales y cerámicas son los bienes materiales más notorios, pero entre las mercancías que no dejan una huella arqueológica tan evidente podemos citar los tejidos, los productos alimenticios e incluso los esclavos. Sin embargo, quizá sea con la cerámica donde esto se pone más de manifiesto.

Los asentamientos fenicios y griegos en el Mediterráneo comenzaron a producir sus propios estilos de alfarería, aunque derivados de los repertorios culturales importados. Así, por ejemplo, en Sicilia, vemos surgir la cerámica sikeliote (griego-siciliana), que se deriva de las tradiciones corintias, áticas y griego orientales (Hodos, 2006; Hodos, 2010: 81-106). En Francia (Massalia), vemos el desarrollo del Cream Ware Colonial (cerámica vidriada en color cremoso) y del Gris Monocromo, el primero de los cuales se convirtió en el estándar de vajilla del asentamiento (Dietler, 2010). Los fenicios de Cerdeña, Sicilia, Malta y Cartago 
compartían un koine de producción regional que se derivaba principalmente del Mediterráneo oriental (por ejemplo, ánforas geométricas bicromáticas, jarras en forma de hongo, jarra con pico en forma de trébol, urnas, tipos de platos) (Hodos in: Knapp e Dommelen, 2014; 214-229). Las características de las cerámicas griegas coloniales fueron incorporadas a las producciones autóctonas. En Sicilia, por ejemplo, comunidades sicilianas rápidamente adoptaron el uso del trébol oinócoe. El trébol siciliano oinócoe de los siglos VI y V continuaron utilizando motivos geométricos derivados de las importaciones de los siglos VIII y comienzos del VII, en lugar de motivos contemporáneos que las propias colonias griegas producían e importaban (Hodos in: Attema, Nijboer e Zifferero, 2005: 103-108).

La tercera es la normalización. Una de las características más notables del primer milenio AEC es la difusión del alfabeto griego en todo el Mediterráneo. Derivado del alfabeto fenicio de finales del siglo IX AEC, los primeros ejemplos de supervivencia del alfabeto griego se asocian a menudo con un sentido de propiedad, mediante letras individuales, inscripciones de dedicatorias y grafitis de posesión (Powell, 2012: 227-244). Los contextos fúnebres y dedicatorios de muchas de estas primeras inscripciones sugieren además que la escritura sirvió a los más diversos fines sociales más allá de la comunicación lingüística directa. Con esto en mente, la propagación de la escritura para los no griegos y no fenicios debe considerarse dentro de un ámbito social.

En Sicilia, la escritura griega es adoptada por primera vez durante el siglo VI por las poblaciones locales para expresar los dialectos hablados, normalmente siguiendo la forma de la colonia griega más cercana (Hodos, 2006: 147-153). Así, las comunidades alrededor de Etna adoptaron la escritura de Catania, al tiempo que los de los Montes Ibleos y alrededor de la llanura de Catania utilizaron la versión en uso en Siracusa y Mégara Hiblea. La escritura de Gela sirvió como modelo a los asentamientos autóctonos en las afueras del centro de Sicilia, mientras que la de Selinute hizo algo muy similar con respecto a las comunidades de Sicilia occidental. La fenicia, utilizada por las colonias fenicias en Sicilia desde el siglo VI, no fue adoptada. Las inscripciones son a menudo poco más que breves expresiones de propiedad o saludo, grabadas en vasos. Esto sugiere una relación explícita entre el consumo de vino y la escritura, siendo que la inscripción tiende a mejorar el estatus del propietario del vaso. El griego se convierte en la lengua de comunicación oficial por el siglo IV. En contraste, en Lattes, Francia, el etrusco se utilizó por primera vez en el siglo VI y el griego solo a partir de la mitad del siglo V. Ninguno de los dos se adaptó

Heródoto, Unifesp, Guarulhos, v.4, n.1 - 2019.1. p.

DOI: https://doi.org/10.34024/herodoto.2019.v4.10088 
más ampliamente para expresar los dialectos locales hasta varios siglos después, cuando el griego cayó en gracia (Dietler, 2010: 70-72).

Es evidente que, más que una rotunda adopción del alfabeto griego y sus funciones, el uso de las letras se modificó para adaptarse a los valores y procedimientos locales. La larga evolución de su adaptación sugiere también que la emulación simple no era el objetivo. En cambio, la naturaleza y el alcance de la utilización del griego y/o del alfabeto griego más allá de los asentamientos coloniales griegos parecen depender, sobretodo, de las circunstancias históricas locales, las particularidades del asentamiento y sus conexiones e interacciones regionales. No refleja un patrón general ni la tasa de desarrollo lingüístico.

El cuarto es la desigualdad. En la Edad del Hierro, ninguna ciudad estado surgió como todopoderosa hasta los siglos VI y V, cuando Cartago se consolida como la base del poder fenicio tras la destrucción de Tiro por los babilonios, a comienzos del siglo VI. Atenas emergió como una fuerza política y económica a tener en cuenta. Sin embargo, uno podría argumentar que las mismas redes de asentamientos fenicios y griegos crearon un medio de comunicación colectivo y la interacción a través de un ámbito más amplio. No fueron dominados por una sola ciudad hasta más tarde, cuando Cartago y Atenas se convirtieron en puntos focales para estas redes comerciales, amalgamando el poder y consolidando los recursos fiscales y de control.

Desde el siglo IX hasta los siglos VI y V, no todas las regiones se relacionaban en forma pareja con estas redes (no significa que estuvieran conectadas por igual después de ese período, pero eso excede el alcance del presente trabajo). Algunas áreas tuvieron vínculos más estrechos que otras en determinados momentos de la Edad del Hierro. Un reciente estudio comparativo de la Bahía de Cádiz, la Bahía de Nápoles, el Golfo de Oristano y el Golfo de León - todos los cuales fueron colonizados por griegos y/o fenicios - revela diversas fechas e índices de conexión entre la región mediterránea más amplia y las regiones del interior a las cuales se accede a través de esos puertos, y que incluso la proximidad de tales conexiones osciló a lo largo del tiempo (Dommelen in: Hodos, 2017: 618633). Se necesita un enfoque local y ascendente para percibir la desigualdad de la visión del mundo globalizado.

El quinto es la homogeneización. La adopción de la cerámica griega, en particular, en las zonas adyacentes a aquellas en las que los griegos fundaron sus asentamientos, y poco después de que los griegos se establecieron en esas áreas (independientemente de las discusiones acerca

Heródoto, Unifesp, Guarulhos, v.4, n.1 - 2019.1. p.

DOI: https://doi.org/10.34024/herodoto.2019.v4.10088 
de las relaciones comerciales anteriores a la creación de estos sitios), así como la rápida imitación de algunas de las formas y motivos por parte de aborígenes alfareros, demuestra un gusto amplio de dichos productos por parte de diversos grupos culturales. Si lo anteriormente expresado también era indicativo de un deseo de ser culturalmente como los productores de estos objetos, la utilización de tales elementos en contextos similares, especialmente domésticos, rituales y funerarios, refleja elementos de prácticas compartidas, ya sean de origen autóctono o adaptativo. Esta combinación del uso de objetos importados y/o imitativos en contextos similares, por lo tanto, presenta un grado de homogeneización en todo el mundo mediterráneo en este momento. Discutiblemente, durante la Edad del Hierro, la distribución y el volumen son desiguales, tanto geográfica como temporalmente, pero puede observarse un amplio grado de similitud. Este uso aparentemente generalizado de las formas de la cultura material griega y en contextos de uso similares es lo que dio lugar a las
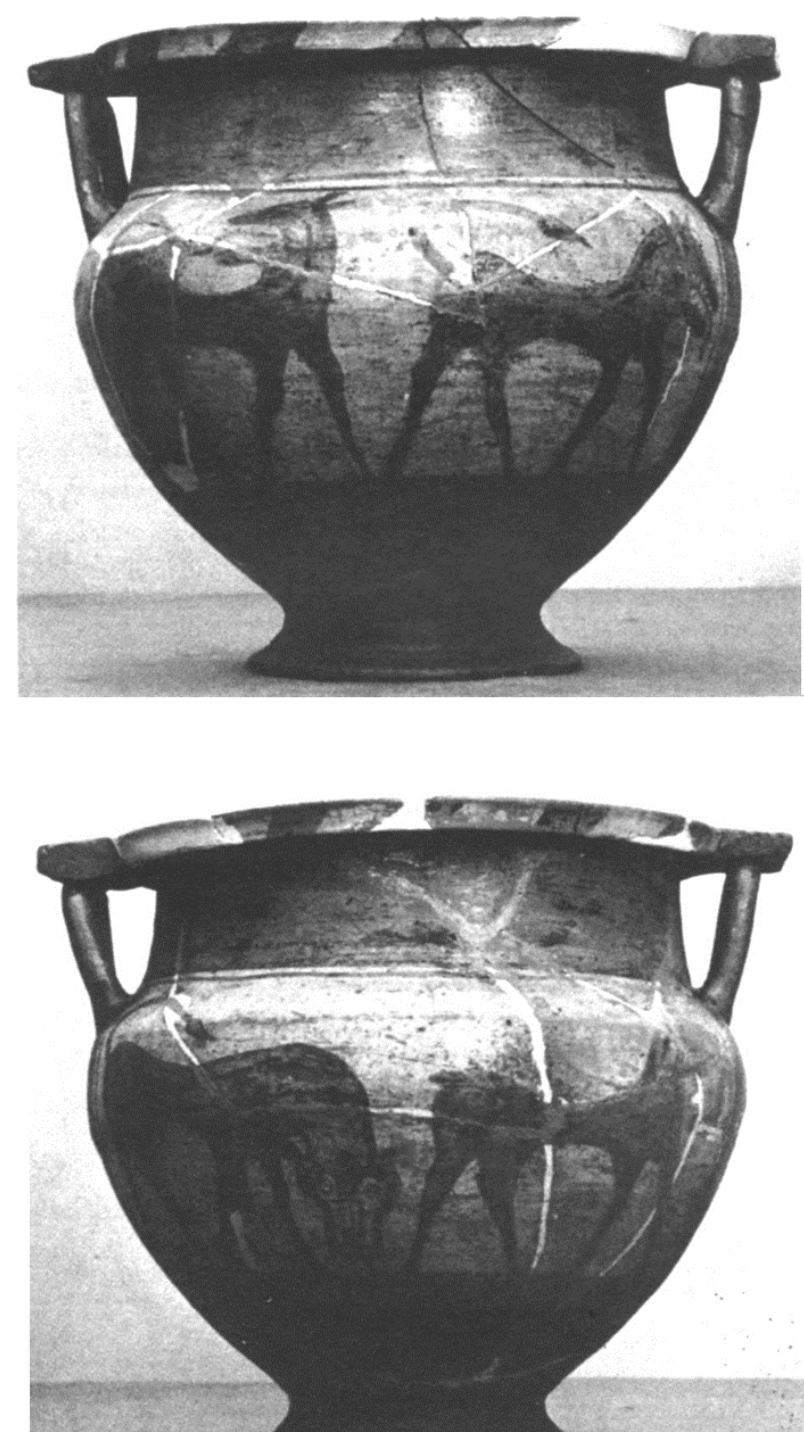

Figura 1. Cratera de Sabucina. ideas de helenización en un principio. La reconstrucción agregada de una serie de asentamientos junto con las formas usadas en los asentamientos griegos (y fenicios) y la adaptación del alfabeto griego por parte de las poblaciones locales refuerza un sentido amplio de la homogeneización en el transcurso de la Edad del Hierro (Cf. Hodos, 2006; Dietler, 2010).

La sexta es la heterogeneidad cultural. Esto se ejemplifica mejor mediante las variabilidades de cerámica que traducen una serie de ideas. Un ejemplo es un krater de Sabucina, un sitio autóctono en el centro de Sicilia (figura 1) (Hodos, 2006: 136-140). La decoración de este vaso en particular representa un par de animales salvajes a ambos lados en un friso. Esta es una reminiscencia de las procesiones

Heródoto, Unifesp, Guarulhos, v.4, n.1 - 2019.1. p.

DOI: https://doi.org/10.34024/herodoto.2019.v4.10088 
de animales heráldicos del período Corintio Medio, pero el ejemplo de Sabucina carece de los ornamentos de llenado y de los detalles incisivos típicos de los productos griegos. El color de la arcilla imita al del Corintio, aunque la forma del cuerpo y pies son, por su forma, del Período Ático. Si bien las importaciones de la cercana colonia griega de Gela y la propia producción de cerámica de Gela pueden haber servido como inspiración, el artista local de Sabucina creó una nueva yuxtaposición, genuinamente local, de ideas.

El séptimo es la reincorporación de la cultura local. Con Sicilia como ejemplo una vez más, la forma arquitectónica doméstica tradicional fue la casa circular (Procelli, 2003). En Sicilia, en el siglo VI, una gran cantidad de emplazamientos sicilianos adoptaron planos urbanos con bloques articulados a lo largo de calles grandes y estrechas, arquitectura rectilínea y murallas que rodeaban la ciudad - características asociadas con el entorno urbano griego y fenicio - . Es notable, por lo tanto, que durante el siglo VI, un gran número de centros autóctonos, a pesar de haber adoptado la arquitectura rectilínea, también mantuvieron grandes estructuras circulares que servían de templo o santuario - una finalidad cultural muy explícita-. Se erigió determinada edificación en Sabucina en ese momento, por ejemplo (figura 2). Lo que es particularmente notable es que había un pórtico in antis, flanqueado por dos columnas para crear un pronaos. Esta es una singular yuxtaposición de tradición siciliana y la

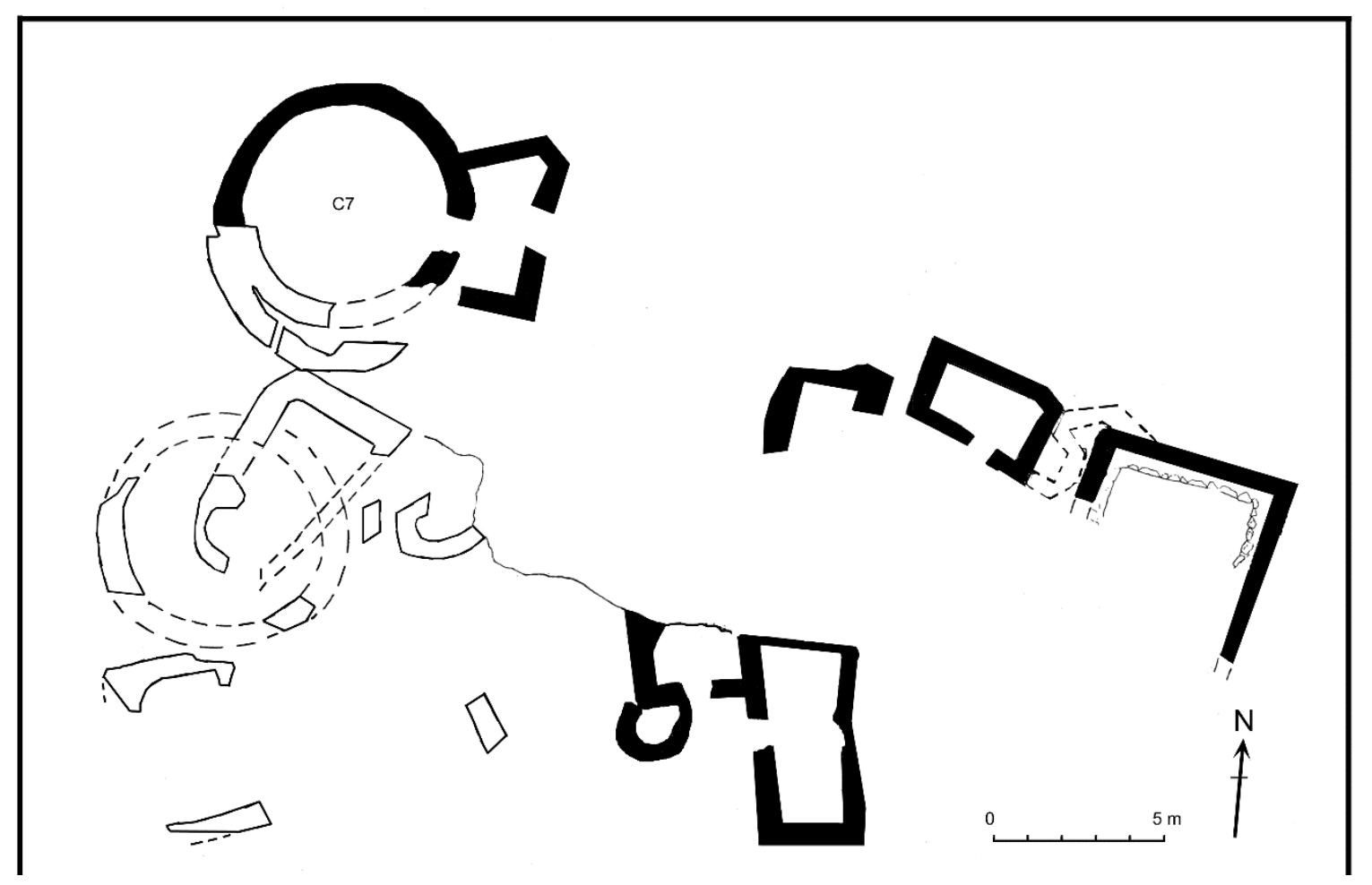

Figura 2. Planta baixa de Sabucina, incluindo a estrutura circular C7.

Heródoto, Unifesp, Guarulhos, v.4, n.1 - 2019.1. p.

DOI: https://doi.org/10.34024/herodoto.2019.v4.10088 
evolución arquitectónica que deriva de formas griegas: se adhiere a las tradicionales formas circulares y se reservaba para los edificios religiosos (en consonancia con la actual práctica de Sicilia), pero al mismo tiempo adopta la noción de un pronaos de las estructuras religiosas griegas (Hodos, 2010: 92-93).

Finalmente, la octava es la vulnerabilidad. La conectividad compleja conduce a la interdependencia debido a las numerosas conexiones, profundamente arraigadas en los diversos grupos. El sistema globalizado creado por las redes de asentamientos griegos y fenicios capturó a las poblaciones locales en sus conflictos mutuos. Por el siglo VI, con la destrucción de Tiro en el Mediterráneo oriental, Cartago tuvo un papel político más abiertamente activo en el Mediterráneo central, surgiendo como el punto focal de la civilización fenicia. Este es el nacimiento de la era púnica de la historia fenicia. Todo comenzó en Sicilia, cuando Cartago obtuvo control sobre las ciudades fenicias de Sicilia occidental. Esto hizo que griegos y cartagineses entraran en conflicto directo unos con los otros. Las poblaciones autóctonas locales quedaron atrapadas en el conflicto, dado que se aliaron con uno u otro bando para obtener el control territorial.

\section{Conclusiones}

En conclusión, podemos afirmar que tenemos todas las características de la globalización moderna en el primer milenio AEC. Pero quizá sea mejor referirnos a una era en vías de globalización, en vez de una era globalizada. Queda claro, a partir de la trayectoria de largo plazo que el pasado nos muestra, que el desarrollo de la globalización en la Edad del Hierro en el Mediterráneo fue un proceso desigual, tanto temporal como espacialmente. Demoró unos buenos cuatrocientos años, del siglo IX al siglo $\mathrm{V}$, para que estas complejas conexiones se desarrollaran plenamente.

Estos procesos comenzaron cuando los griegos y los fenicios crearon infraestructuras de red física vinculadas por la cultura común - sus colonias - y las utilizaron para ampliar la empresa comercial y ejercer influencia política. A través de sus redes mercantiles, las ideas acerca de las formas artísticas y arquitectónicas se difundieron y los mecanismos para facilitar la comunicación pasaron a ser adoptados y adaptados. Esto creó una sensación de compresión tiempo-espacio por medio del sentimiento de pertenencia de la globalización, pero estas adaptaciones fueron desiguales en su adopción a lo largo del tiempo y en todo lugar.

Heródoto, Unifesp, Guarulhos, v.4, n.1 - 2019.1. p.

DOI: https://doi.org/10.34024/herodoto.2019.v4.10088 
¿Cómo esto se compara con otros recientes paradigmas del Mediterráneo, a saber, la conectividad promovida por Horden y Purcell (2000), la perspectiva económica impulsada por el desarrollo, ofrecida por Broodbank (2013), y el enfoque de red de Malkin (2011)?. Los tres comparten un interés común en lo que hace del Mediterráneo un mundo conectado. Para Horden y Purcell, es una combinación de la interacción microrregional, la facilidad de comunicación tanto entre las microrregiones como de larga distancia y la inestabilidad climática y ecológica de la región en general (lo que estimula la sobreproducción y la redistribución). Por lo tanto, ellos ven la interacción dinámica entre la actividad humana y los recursos naturales como la narrativa que sustenta la historia mediterránea. La unidad de esta narrativa radica en la disociación presente en cada uno de los tres aspectos: la fragmentación y la multiplicidad de las microrregiones, la inestabilidad inherente del entorno natural y la variabilidad en la movilidad.

Broodbank amplía esta perspectiva al examinar las cambiantes redes de pueblos alrededor de la región mediterránea. Así, el movimiento de individuos, grupos, objetos e ideas ocupa un lugar central, con las consiguientes competencias e interdependencias económicas y políticas que acompañaron una comprensión cada vez más compartida de los indicadores de riqueza y de estatus, los estilos artísticos e incluso el sincretismo religioso. Este es el caso, en particular, de su discusión sobre la Edad del Hierro.

El énfasis de Malkin está en las redes que sustentan esta conectividad, especialmente dentro del mundo griego. Él demuestra que los conceptos de Grecia y "los griegos" evolucionaron desde las redes de múltiples grupos étnicos, culturales y direccionales de la época, y que fue la circulación y las distancias entre los griegos lo que generó sus similitudes y consolidó sus identidades compartidas en una amplia extensión geográfica.

Las tres perspectivas se ajustan perfectamente en el marco de los estudios de la globalización. Un estudio minucioso de las tres obras y el examen de los ejemplos presentados anteriormente demuestran que la conectividad de la Edad del Hierro tuvo lugar en determinados contextos geográficos y ecológicos. Un enfoque ascendente, por medio de estudios de caso, también pone de relieve el hecho de que las conectividades no se desarrollaron en forma lineal, o a un ritmo uniforme, y tampoco eran estables, al tiempo que su grado se extendió bastante más allá de las zonas litorales del Mediterráneo. Así pues, la globalización es mejor explorada desde dos ángulos: el enfoque ascendente de los estudios de caso

Heródoto, Unifesp, Guarulhos, v.4, n.1 - 2019.1. p.

DOI: https://doi.org/10.34024/herodoto.2019.v4.10088 
individuales y localizados y el nivel descendente de las prácticas compartidas. ${ }^{6}$ Lo que une ambos extremos son las redes de conectividad mediante las cuales los bienes y las ideas se desplazan. Las redes facilitan el desarrollo de prácticas compartidas. Quienes participan en estas redes deberán responder individualmente por su participación; algunos colaboran más estrechamente (en algunos escenarios de conectividad), y otros menos estrechamente, y cada uno de ellos responderá por sus interacciones en la red globalizada de cara a abordar las necesidades y exigencias de su propio sistema sociocultural.

Los núcleos de asentamiento y sus periferias - el tema del presente trabajo - tienen un papel que desempeñar en un sistema globalizado. Las redes apoyan las conectividades de una visión globalizada, pues la noción de red proporciona herramientas para el análisis de conectividades a través del espacio, así como propiedades emergentes de dichas conexiones a lo largo del tiempo (Knappett in: Hodos, 2017: 29-41). Estas redes operan a través de sus nodos, que son los propios emplazamientos, dando así una dimensión física, geográfica. Pero no existe un sistema de interacción igualmente equilibrado. Siempre habrá centros más fuertes y más débiles, así como vínculos más fuertes y más débiles que conecten a los distintos sitios. Aquí es donde la noción de asentamientos y periferias entra en juego en un marco de globalización. Ellos también tendrán una amplia gama de conectividades, cada una de las cuales tendrá altibajos a lo largo del tiempo.

Por estas razones, sostengo que una perspectiva de globalización nos permite recuperar los diversos conjuntos de datos utilizados como base para la promoción de diversas perspectivas, históricamente y en la actualidad. Esto incluye el uso generalizado de la cerámica griega y las formas arquitectónicas, que dieron lugar al modelo de helenización en primer lugar y a la variabilidad dentro de dicho uso, que posteriormente fomentaron las interpretaciones de hibridación. Está claro ahora que existía variabilidad de práctica entre las colonias griegas en sí mismas y las colonias fenicias en sí mismas, que revelan patrones similares. Un marco de interpretación de la globalización posibilita examinar el equilibrio entre los rasgos compartidos que nos permiten hablar de "los griegos" y "los fenicios", al tiempo que nos permite reconocer las diferencias en las prácticas de ser griego o ser fenicio. También podemos reconocer las tensiones encontradas en las prácticas compartidas entre diferentes grupos culturales y las articulaciones de sus identidades sociales, que contrastan

${ }^{6}$ Tal como lo preconiza explícitamente van Dommelen en "Classical Connections," 62829. (Dommelen in: Hodos, 2017: 618-633).

Heródoto, Unifesp, Guarulhos, v.4, n.1 - 2019.1. p.

DOI: https://doi.org/10.34024/herodoto.2019.v4.10088 
con las similitudes de una implicación más amplia. Esto forma parte de la esencia de la globalización. Una perspectiva globalizada también permite que los fenicios y los griegos se reinserten juntos en la narrativa arqueológica del Mediterráneo. Las actividades de los fenicios y sus interacciones con los griegos y otros pueblos en el Mediterráneo son un aspecto tan integral para la comprensión de la relación griegos - no griegos como los griegos lo son con respecto al desarrollo cultural fenicio en el Mediterráneo. Su estudio no debería estar separado por disciplina.

En resumen, las características comunes ampliamente compartidas que se desarrollaron en todo el Mediterráneo durante la Edad del Hierro no reflejan la helenización ni la fenicionización, sino que se entienden ampliamente como "mediterranizaciones" (Morris, 2003: 30-55) templadas por identidades localizadas fuertemente articuladas. Por esta razón, con relación a las complejidades sociales que evolucionaron durante la Edad del Hierro en todo el Mediterráneo, un enfoque de globalización crea una comprensión mucho más rica de lo que hemos podido apreciar antes.

\section{Referencias bibliográficas}

"Globalization Thinking and the Past," em The Routledge Handbook of Archaeology and Globalization, ed. HODOS, Tamar (Londres: Routledge, 2017), 54-65.

AUBET, Marie Eugenia. The Phoenicians and the West: Politics, Colonies and Trade (Cambridge: Cambridge University Press, 2001), 250-56;

BELOCH, Karl Julius. Griechische Geschichte, volume 1.1 (Strassburg: K.J Trübner, 19132), 229-32.

BOARDMAN, John. The Greeks Overseas (Londres: Thames e Hudson, 1999), 38, 190, 268.

BROODBANK, Cyprian. The Making of the Middle Sea (Londres: Thames e Hudson, 2013).

BURKE, Peter. Cultural Hybridity (Cambridge: Polity Press, 2009), 34.

CALDWELL, Melissa. "Domesticating the French Fry: McDonald's and consumerism in Moscow," Journal of Consumer Culture 4.1: (2004) 5-26. 
DIETLER, Michael. Archaeologies of Colonialism. Consumption, entanglement and violence in ancient Mediterranean France (Berkeley e Los Angeles: University of California Press, 2010);

DOMÍNGUEZ, Adolfo. "Local Responses to Colonisation: Some Additional Perspectives," Ancient West and East 11 (2012): 205-18.

DOMMELEN, Peter van. "Classical Connections and Mediterranean Practices," em The Routledge Handbook of Archaeology and Globalization, ed. HODOS, Tamar. (Londres: Routledge, 2017), 618-33.

DOUGHERTY e KURKE. The Cultures Within Greek Culture (Cambridge: Cambridge University Press, 2003);

FEATHERSTONE, Mike. Undoing Culture: Globalization, Postmodernism and Identity (Londres: Sage, 1995), 114.

FRIEDMAN, Jonathan. "Global Crises, the Struggle for Cultural Identity and Intellectual Pork Barrelling: Cosmopolitans versus Locals, Ethnics and Nationals in an Era of Dehegemonisation" in: Debating Cultural Hybridity: multi-cultural identities and the politics of anti-racism, eds. WERBNER, Pnina. MODOOD, Tariq (Londres: Zed Books, 1997), 70-89, 88;

GIDDENS, Anthony. The Consequences of Modernity (Cambridge: Polity Press, 1990);

GIDDENS, Anthony. The Consequences of Modernity (Stanford: Stanford University Press, 1990);

HALL, Jonathan. Hellenicity (Chicago: Chicago University Press, 2002).

HARVEY, David. The Condition of Postmodernity (Oxford: Blackwell, 1989).

HODOS, Tamar, "Stage Settings for a Connected Scene: Globalization and Material Culture Studies in the Early First Millennium BCE Mediterranean". Archaeological Dialogues 21.1 (2014): 24-30, 27.

HODOS, Tamar. "Changing Communities in Iron Age Sicily," em Communities and Settlements from the Neolithic to the Early Medieval Period: proceedings of the $6^{\text {th }}$ Conference in Italian Archaeology, Groningen, April 15-17, 2003, eds. ATTEMA, Peter. NIJBOER, Albert. ZIFFERERO, Andrea. (Oxford: British Archaeological Reports, 2005), 103-08.

HODOS, Tamar. "Colonial Engagements in the Global Mediterranean Iron Age," Cambridge Archaeological Journal 19.2 (2009): 221-41. 
HODOS, Tamar. "Colonisation and Cultural Developments in the Central Mediterranean," em Cambridge Handbook of the Mediterranean World in the Bronze-Iron Ages, eds. KNAPP, Bernard. DOMMELEN, Peter van. (Cambridge: Cambridge University Press 2014), 214-29.

HODOS, Tamar. "Global, Local and In Between: Connectivity and the Mediterranean," no Globalisation and the Roman World, eds. PITTS, Martin. VERSLUYS, Miguel John (Cambridge: Cambridge University Press, 2014), 240-53.

HODOS, Tamar. "Globalization and Colonization: A View from Iron Age Sicily," Journal of Mediterranean Archaeology 23.1 (2010): 81-106. 136-40. 147$53,92-93$.

HODOS, Tamar. "Globalization: Some Basics," em The Routledge Handbook of Archaeology and Globalization, ed. Tamar Hodos (Londres: Routledge, 2017), $3-11,4,5$.

HODOS, Tamar. ed. The Routledge Handbook of Archaeology and Globalization (Londres: Routledge, 2017).

HODOS, Tamar. Local Responses to Colonisation in the Iron Age Mediterranean (Londres: Routledge, 2006).

HORDEN, Peregrine. PURCELL, Nicholas. The Corrupting Sea (Oxford: Blackwell, 2000).

JENNINGS, Justin. "Distinguishing Past Globalizations," em The Routledge Handbook of Archaeology and Globalization, ed. HODOS, Tamar (Londres: Routledge, 2017), 12-28, 14-16.

JENNINGS, Justin. Globalizations and the Ancient World (Cambridge: Cambridge University Press, 2011), 123-41;

KNAPPETT, Carl, "Globalization, connectivities and networks: an archaeological perspective," em The Routledge Handbook of Archaeology and Globalization, ed. HODOS, Tamar. (Londres: Routledge, 2017), 29-41.

KNAPPETT, Carl. EVANS, Tim. RIVERS, Ray. "Modelling Maritime Interaction in the Aegean Bronze Age," Antiquity 82 (2008): 1009-24;

KNAPPETT, Carl. EVANS, Tim. RIVERS, Ray. "The Theran Eruption and Minoan Palatial Collapse: New Interpretations Gained from Modelling the Maritime Network," Antiquity 85 (2011): 1008-23. 
LEIGHTON, Robert. Sicily Before History (Londres: Duckworth, 1999) 17080;

LOMAS, Katheryn. ed. Greek Identity in the Western Mediterranean (Leiden: Brill, 2004);

MALKIN, Irad. A Small Greek World. Networks in the ancient Mediterranean (Oxford: Oxford University Press, 2011).

MOORE, Karl. LEWIS, David. The Origins of Globalization (Nova Iorque e Londres: Routledge, 2009).

MORLEY, Neville. "Globalisation and the Roman Empire," in Globalisation and the Roman World, eds. Martin PITTS e Miguel John VERSLUYS (Cambridge: Cambridge University Press, 2014), 49-68.

MORRIS, Ian. "Mediterraneanization," Mediterranean Historical Review 18.2 (2003): 30-55.

MOSCATI, Sabatino. Il Mondo dei Fenici (Milan: Il Saggiatore, 1966).

MOSCATI, Sabatino. The World of the Phoenicians (Londres: Cardinal, 1968), 77 .

MÜLLER, Christel. "Globalization, Transnationalism, and the Local in Ancient Greece," Oxford Handbooks, 2016, Online em: http:// www.oxfordhandbooks.com/view/10.1093/oxfordhb/978019993 5390.001.0001/oxfordhb-9780199935390-e-42.

PIETERSE, Jan Nederveen. "Rethinking Modernity and Capitalism: Add Context and Stir," Sociopedia Colloquium 1 (2014), 1-11.

PIETERSE, Jan Nederveen. Globalization and Culture (Malden: Rowman e Littlefield, 2015), 7-25, 67, 68.

POWELL, Barry. Writing: Theory and History of the Technology of Civilization (Chichester: Wiley-Blackwell, 2012), 227-44.

PROCELLI, Rosa Maria Albanese. Sicani, Siculi, Elimi (Milan: Longanesi, 2003).

QUINN, Jo Crawley, In Search of the Phoenicians (Princeton: Princeton University Press, 2018), 91-112. 
QUINN, Jo Crawley. "Tophets in the Punic World," em The 'Tophet' in the Phoenician Mediterranean: Studi Epigrafici e Linguistici 29-30 (2012-13), ed. XELLA, Paolo. (Verona: Essedue, 2013), 23-48, 40.

ROBERTSON, Robbie. Three Waves of Globalisation: A history of a development global consciousness (Londres: Zed Books, 2003);

ROBERTSON, Roland. Globalizaton, Social Theory and Global Culture (Londres: Sage, 1992);

SHERRATT, Sue. "A Globalizing Bronze and Iron Age Mediterranean," in: The Routledge Handbook of Archaeology and Globalization, ed. HODOS, Tamar. (Londres: Routledge, 2017), 602-17;

STEIGLITZ, Joseph. Making Globalization Work (Londres: Penguin, 2006), 324,5 .

TOMLINSON, John. Globalization and Culture (Chicago: Chicago University Press, 1992), 2.

TSETSHKLADZE, Gocha. ed., Greek Colonization: An account of Greek colonies and other settlements overseas (Leiden: Brill, 2006).

TSETSKHLADZE, Gocha. "Introduction: Revising Ancient Greek Colonisation," no Greek Colonisation: An Account of Greek Colonies and Other Overseas Settlements, ed. TSETSKHLADZE, Gocha (Leiden: Brill, 2006), xxiii-lxxxiii, xlix $(23-83,49)$

VLASSOPOULOS, Kostas. Greeks and Barbarians (Cambridge: Cambridge University Press, 2013);

WALDBAUM, Jane. "The Coming of Iron in the Eastern Mediterranean," no The Archaeometallurgy of the Asian Old World, ed. PIGGOT, Vincent C. (Filadélfia: Museu da Universidade da Pensilvânia, 1999), 27-57;

WALDBAUM, Jane. From Bronze to Iron: The Transition from the Bronze Age to the Iron Age in the Eastern Mediterranean (Göteborg: Paul Aström, 1978);

WALLERSTEIN, Immanuel. Geopolitics and Geoculture (Cambridge: Cambridge University Press, 1991);

WARD, Kevin. ENGLAND, Kim. "Introduction: Reading Neoliberalization," in Neoliberaliztion: States, Networks, Peoples, eds. WARD, Kevin. ENGLAND, Kim. (Oxford: Blackwell, 2007), 1-22; 
WIJNGAARDEN, Gert Jan van. Use and Appreciation of Mycenaean Pottery in the Levant, Cyprus and Italy (1600-1200 BC) (Amsterdã: Amsterdam University Press, 2002).

YALÇIN, Ünsal. "Early Iron Metallurgy in Anatolia," Anatolian Studies 49 (1999): 177-87. 